\title{
The Change of the Spatial Parameters of the Destruction of the Rock mass by Borehole Charge with Low-Density Tamping
}

\author{
Igor Katanov ${ }^{1, *}$ \\ ${ }^{1}$ T. F. Gorbachev Kuzbass State Technical University, 650000 Kemerovo, 28 Vesennya st., Russian \\ Federation
}

\begin{abstract}
Explosive destruction of the rock formation is substantiated by several theories developed by well-known scientists. The improvement of quality of preparation of rock mass to excavation, by an excavator without an increase in the value of specific consumption of explosives is important in the present time. Traditionally, to increase the impact time of detonation products on the rock hard tamping was used. The problem is in the rational redistribution of the explosion energy due to the use of a borehole charge, and in particular, in the tamping of low-density, porous materials. The more intensive attenuation of the mass velocity of particles in the material of such tamping in comparison with the mass velocity of the rock mass particles contributes to the well channel compression and increases the impact time of detonation products on the rock mass. As a result of redistribution of energy of detonation products, the specific impulse of explosion increases. The value of the radius of the controlled crushing zone increases by more than 1.6 times. The results of industrial explosions in coal mines have confirmed the theoretical reasoning.
\end{abstract}

\section{Introduction}

One of the important directions of improvement of blasting operations is to increase the efficiency of the explosion as a result of rational redistribution of the share of explosive energy in time and space. The use of modern explosives in traditional methods of blasting operations is accompanied by significant refinement of the rock mass in the near-to-charge zone, which at the same time becomes an area of a strong energy absorption. These large energy losses at the initial stage of development of the explosion cannot be subsequently compensated.

When crushing rock mass, it is necessary that the main compression wave had amplitude above the rock strength limit. By changing the parameters of the location of borehole charges and their design in the rock mass, it is possible to change the distribution of energy in the exploding array rationally. A big role on the quality of rock breaking is a consequence of the redistribution of energy of borehole charges of the material of the tamping.

M. A. Cook $[1,2]$ points to existence of three basic theories of explosive destruction of rocks by explosion. The first theory is the shock wave theory or the theory of funnel formation. The main elements of the shock-wave theory are the impedance of explosives and rocks, which determine the role of reflected waves in the mechanism of destruction [3-5]. The second theory of radial fracture formation was developed by a group of Swedish scientists, in which the shear wave at the early stage of shock wave propagation and the subsequent development of radial cracks

${ }^{*}$ Corresponding author: kib.rmpio@kuzstu.ru 
play a decisive role in the fracture mechanism [6]. M. A. Cook believes that this theory is also a shock-wave one, although the role of the breakdowns in the free surface in it is reduced to a minimum. The third theory is known as the energy theory [7]. According to this theory, the pressure of detonation products compresses the explosive array, bringing it into a tense state, facilitating subsequent crushing, and creating a velocity field in the array, conducive to the start time of the final stage of primary crushing.

Deeply and physically reasonable qualitative picture of the impact of the explosion on the natural surroundings is described in scientific works by F. A. Baum, G. I. Pokrovskyi, O. E. Vlasova, A. N. Khanukaev. M. A. Sadovskyi etc They consider the position of self-similarity of shock wave spread at the explosion and the main energy dependences, establish a number of parameters of the shock wave arising in the natural surroundings at the explosion of the explosive substance.

The analysis of scientific information shows that the ideas of different authors about the mechanism of destruction of the rock explosion, taken separately, do not give a full explanation of this complex physical process. It is impossible to give preference to this or that opinion as each of them to a greater or lesser extent corresponds to certain mining engineering conditions.

\section{Results}

The analytical description of the processes occurring in the detonation of explosive substances in the rock mass and to simplify the solution of the equations of the shock waves spread, usually taking the assumption about the coincidence of the isotherm, isentrope and shock adiabats. To determine the initial voltage at the boundary "explosive substances natural substances" the equations of state of explosives, natural substances, the laws of conservation of mass, driving force (momentum) and energy are used. The action of stress waves is considered in an acoustic approximation. In this context, the explosive effect of the explosion is proportional to the total momentum. Controlling of the momentum components (with voltage $o$ and time $t$ ) allows you to change the result of the destruction of the substances. Almost any material used as a tamping is a substance with different contents of individual components, i.e. air $\left(a_{1}\right)$, liquid $\left(a_{2}\right)$ and solid substance $\left(a_{3}\right)$. If we assume that $\mathrm{a}_{3}=$ 0 , the mixture is a liquid bubble substance. The density of a multicomponent compound is determined by the sum of the products of the density and volume content of each component. Solid tamping (removed particles, drilling detail) contains a minor volumetric concentration of air ( $a_{1}=0,01-0,1$ share units) and therefore its density is from 1.6 to $2.0 \mathrm{~g} / \mathrm{cm} 3$ and tamping on a liquid basis at changing the volume of the air concentration $\left(a_{1}\right)$ is from 0.05 to 0.95 share units the density of the mixture decreases from 0.92 to $0.06 \mathrm{~g} / \mathrm{cm} 3$. It was found [8-9] that viscous properties can have a noticeable effect on the laws of the explosive waves spread and on the intensity of their fading with distance, and mechanical air foam can be compressed under the influence of a shock wave with the absorption of its energy and adjustment of the cellular structure [10-16].

This determines the prospects of its use as a bottom hole in the construction of borehole charges. If the displacement speed of the particles of tamping is commensurate with the mass speed of the particles of the destroyed array, and it is thrown out of the wellhead before the time of cracking, the total time of exposure to detonation products (DP) in the rock mass will be defined by the amount which represents the time of detonation of the charge, and the compression resistance of the material of the tamping of detonation products prior to their dissipation to the atmosphere.

With the increase in the amount of clamped air, the compression time of such tamping, and, consequently, the time of pressure rise and the total time of its impact on the array increases. The build-up of the pressure of detonation products in the well is determined by the time, which takes into account the detonation time of the explosive charge and the time of 
compression of the air in the bottom material under the influence of a plane shock wave. The total time of exposure of detonation products to the rock mass also takes into account the time of locking the detonation products before their dissipation to the atmosphere. The locking time of the detonation products will be:

$$
t_{z}=\left[r_{1} \overline{r_{1}} / \int_{0}^{\overline{r_{1}}} v_{1}(\bar{r}) d r\right]-\left[r_{0} \overline{r_{0}} / \int_{0}^{\overline{r_{0}}} v_{1}(\bar{r}) d r\right],
$$

here $\bar{r}_{0}, r_{0}-$ accordingly, the relative length (radii power) and plot length of tamping compression, at which equalization of the mass speed of the particles of the destroying rock mass and the tamping occurs, $\mathrm{M}$;

$\bar{r}_{1}, r_{1}$ - accordingly, the relative length (in radius of the charge) and the length of the area of well cavity compression by particles of the destroying rock mass, m;

$v_{1}$ - the mass flow rate of particles of rocks, $\mathrm{m} / \mathrm{s}$.

The result of the impact of the shock wave is the displacement of rock particles and tamping. On the interval plot of the compression of the tamping mass speed of particles of rocks and low density tamping are balanced with the subsequent compression of the cavity well and the average displacement speed. With the increase in the amount of clamped air in the material of tamping, the rise time of the detonation product pressure and the total time of its impact on the rock mass increases taking into account the formula (1). Then the total blast impulse $I$, which is described by an exponential dependence, takes the form:

$$
I=\int_{0}^{t_{\mathrm{B}}} P_{\mathrm{H}}\left[\left(\frac{t}{t_{\mathrm{H} 1}}\right)^{\frac{2}{3}} e^{1-\left(\frac{t}{t_{\mathrm{H} 1}}\right)^{\frac{2}{3}}}\right] d t,
$$

here $P_{\mathrm{H}}-$ the initial product detonation (PD) pressure, $\mathrm{Pa}$;

$t_{\mathrm{H} 1}-$ - pressure rise time, $\mathrm{s}$;

$t_{\mathrm{B}}-$ the exposure time of the product detonation (PD), s;

$t$ - time, s.

Analysis of the dependences (1) and (2) shows that the faster the well will begin to break and the longer the locking time of the detonation products in the cavity of the well, the greater the full impulse of the explosion, and, consequently, the explosive action of the detonation product.

\section{Discussion}

For rocks of medium strength and blockage, constituting more than $40 \%$ of covering in the Kuzbass sections and worked out by transport technology, at an average height of the escarpment of $15 \mathrm{~m}$, well depth of $16 \mathrm{~m}$ and their diameter of $0.214 \mathrm{~m}$, the height of the charge column of the standard explosives (grammonite 79/21) is $9.6 \mathrm{~m}$, and the line of the lowest resistance is $5.6 \mathrm{~m}$. As shown by calculations to determine the spatio - temporal parameters of the destruction of covering escarpment by borehole charge with different tamping material, the change of the maximum mass displacement speed of the particles (taking into account the degree of attenuation) of the destructible rocks, hard tamping and foaming-and-gel tamping is significantly different. During detonation of the explosive charge (about 2.7 10‘3) time compression of the material of the solid tamping is $0.0210^{`} 3$ and release it out of the hole the total exposure time of the product detonation pressure on the rock mass is approximately $0.01 \mathrm{~s}$. The release time of the solid tamping is $0.006 \mathrm{~s}$, which is almost 10 times faster than the low-density, porous tamping. When using foam and gel as a tamping, the time of its compression, depending on the volumetric content of the air, $a_{l}$ is 
from 0 to 0.9 share units, increases by 3-4 times compared to the hard bottom.

The locking time of product detonation varies in the range of 0.009-0.023 s., and the total exposure time increases by 2-2.5 times. The speed equalization occurs at a distance of 2-3 radii from the border section "explosive substances-tamping" (Fig. 1).

The curve of the displacement of particles in the rock mass relatively to foam and gel tamping determines the clamping of a borehole by the products of destruction of rocks, an amount equal to 20-30 radii of the charge.In accordance with the parameters of shock waves in the rock mass, the speed of development of the zone of compression of the borehole is about $300 \mathrm{~m} / \mathrm{s}$. With further increase of $a_{1} \rightarrow 1.0$ share units the total time of the detonation product pressure exposure is reduced to a minimum, which in real conditions leads to the "detonation attenuation" of the drill holes. As a result of theoretical research of interaction of physical phenomena occurring during the explosion of the explosive charge in the rock mass and the tamping, methodical approach was formulated. It proves the qualitative change of the space-time parameters that affects the destruction of the rock in a zone adjacent to an uncharged explosives of the well when construction of the charge with the tamping that has variable structural characteristics.

\section{Conclusion}

The analysis of the calculation results shows that the time of the explosion impulse on the rock mass corresponds to the time of discharge of the hard tamping and the hydraulic tamping. And this happens 3 times faster than cracks are formed along the line of the least resistance. Low-density, porous tamping with increasing air content in the range from 0 to 0.9 share units increases the locking time of detonation products.

When the pulse is determined by the formula (2), and equals to $2.05 \cdot 10^{7} \mathrm{~Pa} \mathrm{~s}$, from the explosion of a charge with a low-density explosive substances, with porous bottom containing 0.9 units of air, the radius of the zone of crushing in comparison with the similar explosion of the charge with the solid or hydro tamping having a momentum of $7.5-10^{6} \mathrm{~Pa}$ increases in 1.64 times.

Thus, with general preconditions for increasing the high-explosive effect of the borehole charge due to the locking action of a low-density, porous (for example, foam and gel) tamping compared to solid tamping (for example, drilling fines) are the ability to compress bubble mixtures and a more intense decay of the mass speed of its particles compared to the mass speed of the rock mass. It contributes to the well channel compression and increases the time of the product detonation impact on the rock mass, providing an increase in the specific impulse, and, consequently, the size of the zone of adjustable crushing radius.

\section{References}

1. M. A Cook, The Science of High Explosives (Reinhold Publishing Company, Chicago, 1958).

2. The Science of Industrial Explosives. M. A. Cook Founder and Chairman of the board Ireco chemicals (Motmon Press, Salt Lake City, 1974)

3. K. Hino, Quarterly of the Colorado School of Mines, 51(3) (1956)

4. K. Hino, Journal of the Industrial Explosives Society, 18(4) (1957).

5. T. Sakurai, Journal of the industry. Explosives, 20(1) (1959)

6. J. M. Walsh and R. H. Christian, Phys. Rev. 97, 154 (2000)

7. K. Hino, Journal of the Industrial Explosives Society, 18(1), 345-351 (1997)

8. G. Duvall, Propagation of Plane Shock Wales in a Stress-Relaxing Medium in Stress waves in Anelastie Solids, (Springer Verlag, Berlin, 1964) 
9. J. S. Krasinski, A. Khosla, 5th Austral. Conf. Hydraul. and Fluid Mech., Cristchurch, 1 500 (1974)

10. R. Raspet, P. Butler, F. Yahani, Appl. Acoust, 22(3), 243 (1987)

11. R. Raspet, S. Griffits, J. Acoust. Soc. Amer, 74(6), 1757 (1983)

12. J. Rowers, H. Krier, UILD ENG, 84, 4014 (1984)

13. T. D. Panczak, P. B. Butler, H. Krier, J. Haz. Mater, 14(4) 321 (1987)

14. E. D. Evans, D. F. Jancovski, E. D. Hirlemen, TRS R-78050 (1979)

15. J. Rowers, H. Krier, J. Haz. Mater, 13(1), 121 (1987)

16. P. R. Clark, P. J. Hubbard, P. R. Lee, H. C. Woodman, NSWC-WO, TR 77-36, (1977) 